\title{
EL CARÁCTER CONVENCIONAL DEL DERECHO
}

Federico J. Arena *

* Universidad de Génova, Italia
A veces se sostiene que el convencionalismo acerca de un cierto fenómeno es el punto de vista según el cual ese fenómeno, a pesar de las apariencias, surge de, o es determinado por, una convención. Quizás puede afirmarse que en sede iusfilosófica se ha dado la situación inversa, es decir, aun cuando el derecho parece ser un fenómeno convencional no ha resultado sencillo explicar con precisión en qué consiste este rasgo. El convencionalismo jurídico suele asociarse con quienes responden afirmativamente a las siguientes preguntas: ¿existe una convención entre los jueces?, ¿es esta convención el hecho jurídico fundamental?

Sin embargo, para comprender en qué consiste responder 
afirmativamente, quizás sea adecuado distinguir los distintos ámbitos de lo jurídico que han sido considerados convencionales. Por un lado se sostiene que el concepto mismo de derecho es convencional, en oposición a puntos de vista realistas o escépticos (Nino, Bayón). Por otro lado el convencionalismo ha sido sostenido con relación a la tesis de las fuentes sociales, ya sea como un elemento más primitivo de esa tesis (Coleman, Bayón), ya sea como una especificación de ella (Coleman, Postema, Marmor).

El convencionalismo acerca del concepto de derecho es una posición que se deriva de un modo de concebir los conceptos en general. Según este punto de vista los conceptos no dependen de otra cosa más que de nuestras estipulaciones o de nuestras prácticas, y si bien se acepta que quizás existan algunas excepciones, como los conceptos de clases naturales o los conceptos primitivos, el derecho no formaría parte de ellas. La mayor virtud de este modo de entender el concepto de derecho parecía consistir en su capacidad para disolver las (ahora pseudo) disputas teóricas, transformándolas en elecciones de un concepto en lugar de otro. Sin embargo, este terminó siendo, como veremos, el flanco hacia el que apuntó una de las críticas más persistentes al convencionalismo.

El convencionalismo con relación a la tesis de las fuentes sociales ha sido, como adelanté, desandado siguiendo dos caminos distintos. Uno de ellos ha consistido en sostener que la tesis de las fuentes sociales, según la cual el contenido y la existencia del derecho dependen de hechos sociales, admite un análisis reduccionista en términos de tesis más primitivas, a saber, la tesis convencionalista y la tesis de las fuentes.

Según la tesis convencionalista la identificación de los criterios de validez jurídica es una cuestión que depende de las convenciones existentes al respecto. Según la tesis de las fuentes el contenido de los criterios de validez jurídica (identificados convencionalmente) no tienen que ver con el valor de la norma, sino con su origen. Este modo de descomponer la tesis de las fuentes sociales, distinguiendo entre identificación y contenido de los criterios de validez jurídica, persigue encontrar en la tesis convencionalista el contenido mínimo que compartirían tanto el denominado positivismo excluyente 
como el denominado positivismo incluyente. Y pretende además, como se verá más adelante, ser un modo de afrontar un insistente problema para el convencionalismo. El problema vinculado con el hecho de que los jueces, tal como se asume en el caso, suelen apartarse del significado de las disposiciones normativas cuando, para justificar su decisión, identifican el derecho.

El otro camino del convencionalismo con relación a la tesis de las fuentes sociales es aquél que se propone como una especificación de ésta tesis. En este caso el convencionalismo sostiene que la práctica judicial que fija los criterios de validez jurídica, denominado por Hart regla de reconocimiento, puede ser mejor precisada en términos de una especial convención (de coordinación o constitutiva) entre los jueces. Este es un hilo de discusión que se inicia a partir del presunto giro convencionalista que habría dado Hart en el Postscript a The Concept of Law publicado en 1994, y mediante el cual procuraba responder a sus críticos.

Estos distintos ámbitos del convencionalismo terminan superponiéndose cuando aparece la pregunta acerca de cuál es el concepto (convencional) de derecho presente en la práctica (convencional) de los jueces. En este sentido el convencionalismo ha debido enfrentarse a varias dificultades. Por un lado el convencionalismo conceptual ha debido vérselas con las derivaciones de los argumentos que apuntaron a negar la posibilidad de un fundamento convencional de la lógica (Quine). Estos argumentos repercutieron no sólo en la filosofía de la lógica, sino también en teoría política, en filosofía del lenguaje y en filosofía del derecho. Traducida a la discusión acerca de conceptos, la objeción señalaría que no es posible que todos nuestros conceptos estén basados en convenciones, pues para ello sería necesario un acuerdo entre los poseedores de los conceptos, y un acuerdo tal no puede ser alcanzado sin usar ya ciertos conceptos (por ejemplo el de acuerdo).

D. Lewis mostró que una de las premisas en las que se basa el argumento de Quine, i.e. que la existencia de una convención depende de un acuerdo, es falsa. Ello en cuanto una convención puede surgir sin acuerdo en los casos en que se dan ciertas condiciones que definen un problema de coordinación. Un problema de coordinación se presenta en situaciones en las que encontrándose disponibles dos o más opciones de 
acción, la elección de una de ellas por parte de un individuo depende de la decisión que tomen al respecto otros individuos, y viceversa, y además entre los individuos involucrados existe una cierta coincidencia de intereses. Cuando el problema de coordinación sea recurrente, la solución alcanzada en casos anteriores generará entre los individuos involucrados expectativas recíprocas de comportamiento que los llevarán a converger regularmente en esa solución. Habrá surgido así una convención. En virtud de esta vinculación con los problemas de coordinación estas convenciones han sido posteriormente denominadas convenciones de coordinación, con el objetivo adicional de resaltar la existencia de otros tipos de convenciones.

Más allá del éxito o fracaso del intento de Lewis de rehabilitar el convencionalismo con relación al lenguaje y a la lógica, si su abordaje de las convenciones es plausible entonces es posible que el concepto de derecho judicial sea convencional aun cuando no exista entre los jueces un acuerdo sobre él. Pero una vez descartado el acuerdo se vuelve indispensable ofrecer un relato acerca de cómo la práctica judicial da lugar a la convención. Algunos filósofos del derecho (Postema, Coleman) creyeron en un primer momento encontrar en las convenciones de coordinación una especificación de la tesis de las fuentes sociales que ofreciera ese relato y que además permitiera responder a varias de las objeciones que habían sido dirigidas contra la teoría de las reglas sociales como práctica defendida por Hart. Objeciones tales como la inexistencia de acuerdo, la necesidad de explicar la normatividad del derecho, esto es, su capacidad para ofrecer razones para la acción, y la necesidad de dar cuenta de la autoridad del derecho. Sin embargo parece ser un problema cerrado que recurriendo a este tipo de convenciones no es posible dar una respuesta completa a esos problemas (Green, Dickson, Celano). A pesar de ello, me parece, debe mantenerse abierta la cuestión acerca de cuál sea el lugar (centralidad, relevancia) de estos problemas dentro de un abordaje convencionalista del derecho. De todos modos, las convenciones de coordinación, caracterizadas por la elección arbitraria entre dos opciones, no parecen encajar bien con ciertos rasgos que se atribuyen a la práctica jurídica, sobre todo la importancia política de la elección de los criterios de validez jurídica. El abandono de esta vía no quiere decir 
que las convenciones de coordinación hayan sido olvidadas por los filósofos del derecho, subsisten aún vinculadas a la explicación de algunas de las funciones de la autoridad (Raz) o como explicación de las costumbres jurídicas (Celano).

Estas insuficiencias llevaron a algunos filósofos del derecho a buscar alternativas. Así se propusieron tanto las convenciones constitutivas (Marmor) como las convenciones profundas (Marmor, Bayón).

Mediante las convenciones constitutivas se pretende suplir las deficiencias del abordaje lewisiano, al menos como enfoque de las convenciones en general. Si bien el enfoque de Lewis funciona en algunos casos, el derecho no parece ser uno de ellos. Es decir, este segundo tipo de convenciones se propone, no como alternativa, sino como complemento de las convenciones de coordinación. Por un lado independizando la noción de convención de la de problema de coordinación, por otro lado insistiendo en la capacidad del derecho para definir y crear (hacer posibles) nuevas formas de comportamiento.

Ahora bien, aun si aceptamos la plausibilidad de un enfoque según el cual la existencia de una convención es independiente de la existencia de un acuerdo, en el caso de la práctica judicial parecen darse dos circunstancias que dificultan afirmar que encaja en alguna de las reconstrucciones propuestas. Por un lado, como adelanté, los jueces suelen apartarse del significado convencional de las disposiciones normativas. Por otro lado suelen desacordar acerca del contenido del derecho y acerca de los criterios para identificarlo.

Con relación al primero de los hechos el convencionalista podría optar por ceñir su pretensión y sostener que la convención alcanza sólo la identificación de las disposiciones normativas. Creo que es un problema abierto si una convención así restringida, i.e. que se limita a las disposiciones y no incluye su significado, es una idea plausible.

Otro intento de dar cuenta del primero de estos hechos consistió en ampliar el conjunto de hechos sociales relevantes. En este sentido, junto a las convenciones semánticas que fijan el significado literal de los textos jurídicos, se postula también la existencia de convenciones interpretativas que fijan métodos de interpretación 
adicionales. De este modo, del hecho de que los jueces se aparten del significado literal no se sigue todavía que para la identificación del derecho abandonen las convenciones y se aboquen a argumentos morales, sino que puede tratarse todavía de convenciones interpretativas.

Sin embargo esta extensión cuantitativa de la convención fue considerada insuficiente. Para dar cuenta del hecho en cuestión resultaría necesario en cambio articular de modo más complejo el contenido mismo de tales convenciones. Esta articulación exigiría abandonar la tesis de las fuentes para defender aisladamente la tesis convencionalista. Es decir, se trataría de sostener que si bien cuáles sean los criterios de validez es una cuestión que depende de convenciones (tesis convencionalista), los criterios así individualizados no han de referirse necesaria y únicamente a hechos sociales, sino que pueden referirse también al valor o corrección moral de la norma (negación de la tesis de las fuentes). Esta propuesta teórica, que caracteriza a los defensores del positivismo incluyente y que pretende dar un espacio a los argumentos morales dentro del convencionalismo, debe enfrentarse a lo que podemos denominar los dos dilemas de Bayón.

El primer dilema se presenta cuando se intenta especificar el contenido de los criterios de validez jurídica que han sido identificados convencionalmente. Existen dos opciones, o bien el contenido de esos criterios depende a su vez de convenciones, i.e. de lo que los jueces creen que es moralmente correcto, pero en este caso se excluye la argumentación moral sustantiva y se termina otra vez comprometido con la tesis de las fuentes; o bien esos criterios no son convencionales, pero en este caso la única opción disponible es que se trata de criterios morales objetivamente correctos.

Para evitar el primer cuerno, el defensor del positivismo incluyente bien podría asumir un punto de vista realista acerca de la moral y sostener que se trata de una convención de seguir criterios moralmente correctos. Se enfrentaría así al segundo dilema. O bien existe acuerdo acerca de cuáles son los criterios moralmente correctos, pero entonces se regresa nuevamente a la tesis de las fuentes; o bien no existe acuerdo, pero entonces se trata de una convención de seguir criterios no convencionales. De este modo, ambos dilemas comparten un 
cuerno, a saber, el verse inevitablemente comprometido con la tesis de las fuentes, y se diferencian en el segundo cuerno. Mientras en el segundo cuerno del primer dilema acecha el realismo moral, en el del segundo dilema acechan las convenciones huecas o aparentes. Para escapar también a este segundo dilema de Bayón, el positivista incluyente debería volver plausible la noción de "convención de seguir criterios no convencionales", i.e. convenciones para cuya existencia basta que la convergencia se verifique sobre cuáles son los criterios a seguir, aun cuando no exista un acuerdo acerca del contenido de tales criterios (Coleman). Que esta no es una buena estrategia parecía ser un problema cerrado (Bayón), aunque existe un intento reciente de rehabilitarla (Orunesu).

A pesar de estos esfuerzos, la cruz del convencionalismo sigue siendo su dificultad para ofrecer criterios sustantivos que permitan elegir entre conceptos diferentes y que permitan otorgar relevancia a las discusiones sobre ellos. Un convencionalista convencido debería responder que se trata sólo de estipulaciones distintas o prácticas divergentes. Si bien esta actitud parecía adecuada para cuestiones terminológicas, algunos teóricos (Dworkin) sostienen que esto es incompatible con el modo en el que entendemos ciertas cuestiones jurídicas en las que se encuentran involucradas nuestras más profundas convicciones. Y este sigue siendo uno de los problemas abiertos para el convencionalismo, es decir, si puede volver compatible la afirmación de que el concepto de derecho depende de nuestras estipulaciones o prácticas con la posibilidad de que existan desacuerdos sustantivos acerca de ese concepto.

El problema de los desacuerdos teóricos es uno de los problemas del convencionalismo que más atención ha recibido en los últimos años e incluso ha sido ya abordado en esta misma sección de Discusiones. Mediante las convenciones profundas, finalmente, se persigue resolver este problema otorgando un espacio conceptual a la posibilidad de que los criterios de validez jurídica sean convencionales aun cuando no exista acuerdo acerca de ellos o resulten opacos a los individuos que forman parte de la 
convención. Al menos si este fenómeno se produce hasta un cierto nivel.

Que ambas estrategias, aquella basada en la idea de convenciones constitutivas y aquella basada en la idea de convenciones profundas, sean viables, es todavía un problema abierto.

Puede agregarse, para concluir, que los defensores del convencionalismo jurídico se han preocupado sobre todo por responder a cada una de las críticas que fueron dirigidas al abordaje hartiano del concepto de derecho. Quizás pueda afirmarse que este punto de vista acerca del derecho se ha encontrado en una posición defensiva, que incluso parece haber conducido al fracaso. Este fracaso provoca que no sean pocos los que dudan o niegan que el derecho efectivamente sea un fenómeno convencional.

Sin embargo quizás sería también una cuestión abierta determinar qué aspecto adquiriría la discusión si se insistiera en cambio en las virtudes de un abordaje convencionalista del derecho. 